\title{
Study of the influence of long-term high-temperature operation on the structure and properties of austenitic chromium-nickel-molybdenum steel
}

\author{
K. A. Okhapkin ${ }^{\dagger}$, A.S. Kudryavtsev \\ †kirill.okhapkin@mail.ru \\ NRC “Kurchatov Institute” - CRISM “Prometey”, Saint Petersburg, 191015, Russia
}

\begin{abstract}
The effect of long-term operation at a temperature of $515^{\circ} \mathrm{C}$ on the structure and properties of steel $08 \mathrm{Cr} 16 \mathrm{Ni1} 1 \mathrm{Mo} 3$ (grade 316) is studied. Data on the structure and phase composition of the steel were obtained using optical and scanning electron microscopy. The phase composition of steel in the equilibrium state was determined by thermodynamic modeling in the FactSage software package. As a result of the study, it was found that there are the structure changes with the formation of secondary phases initiated by the precipitation of elements with limited solubility from the supersaturated solid solution during operation at a temperature of $515^{\circ} \mathrm{C}$ for 195000 hours in steel $08 \mathrm{Cr} 16 \mathrm{Ni11Mo3}$ (grade 316). The presence of the following secondary precipitates in the structure of the solid solution of austenite was revealed: carbide of the $\mathrm{M}_{23} \mathrm{C}_{6}$ type $(\mathrm{M}-\mathrm{Cr}, \mathrm{Mo})$, ferrite $(\alpha)$, intermetallic phases. Based on a comparison of the thermodynamic modeling data and the experimental determination of the phase composition, it was found that the structure of steel is close to equilibrium. The results of static tensile tests have shown that a change in the phase composition during heat aging leads to embrittlement of steel, i.e. a decrease in ductility. In general, the mechanical properties of the material in the state after operation are close to the original ones. The tensile strength at room temperature is $5 \%$ higher than the initial one, and in the range of elevated temperatures of $350-600^{\circ} \mathrm{C}$ it is $5-10 \%$ lower than the initial level. The yield strength at temperatures of 20,500 and $600^{\circ} \mathrm{C}$ after operation increases by $5-10 \%$ of the initial level, and at temperatures of 350 and $550^{\circ} \mathrm{C}$ it practically does not change. In the temperature range $350-600^{\circ} \mathrm{C}$, the ductility values of steel $08 \mathrm{Cr} 16 \mathrm{Ni} 11 \mathrm{Mo} 3$ (grade 316 ) decrease by $5-17 \%$ from the initial level. The main contribution to the change in the mechanical characteristics of steel is made by secondary precipitates of carbide and intermetallic phases.
\end{abstract}

\section{Исследование влияния длительной высокотемпературной эксплуатации на структуру и свойства аустенитной хромоникельмолибденовой стали}

\author{
Охапкин К. А. ${ }^{\dagger}$, Кудрявцев А. С. \\ НИЦ «Курчатовский институт» - ЦНИИ КМ «Прометей», Санкт-Петербург, 191015, Россия
}

В работе исследовано влияние длительной эксплуатации при температуре $515^{\circ} \mathrm{C}$ на структуру и свойства стали марки 08X16H11M3. Данные по структуре и фазовому составу стали получены с помощью оптической и растровой электронной микроскопии. Фазовый состав стали в равновесном состоянии определен путём термодинамического моделирования в программном пакете FactSage. В результате исследования установлено, что в процессе эксплуатации при температуре $515^{\circ} \mathrm{C}$ продолжительностью 195000 ч в стали марки 08X16Н11M3 происходит изменение структуры с образованием вторичных фаз, инициированное выделением элементов с ограниченной растворимостью из пересыщенного твердого раствора. Обнаружено присутствие в структуре твердого раствора аустенита следующих вторичных выделений: карбид типа $\mathrm{M}_{23} \mathrm{C}_{6}(\mathrm{M}-\mathrm{Cr}, \mathrm{Mo})$, феррит ( $\alpha$ ), интерметаллидные фазы. На основании сопоставления данных термодинамического моделирования и экспериментального определения фазового состава, установлено, что структура стали находится в состоянии близком к равновесному. Результаты испытаний на статическое растяжение показали, что изменение фазового состава в процессе теплового старения приводит к охрупчиванию стали - снижению пластичности. В целом, механические свойства материала в состоянии после эксплуатации близки к исходным. Временное сопротивление при комнатной температуре на 5\% выше 
исходного, а в диапазоне повышенных температур $350-600^{\circ} \mathrm{C}$ на 5-10\% ниже исходного уровня. Предел текучести при температурах 20,500 и $600^{\circ} \mathrm{C}$ после эксплуатации повышается на $5-10 \%$ от исходного уровня, а при температурах 350 и $550^{\circ} \mathrm{C}$ практически не изменяется. В диапазоне температур $350-600^{\circ} \mathrm{C}$ значения пластичности стали марки 08X16Н11M3 снижаются на 5-17\% от исходного уровня. Основной вклад в изменение механических характеристик стали вносят вторичные выделения карбидных и интерметаллидных фаз.

Ключевые слова: парогенератор, сталь, эксплуатация, термическое старение, свойства.

\section{1. Введение}

Аустенитная хромоникельмолибденовая сталь марки 08X16Н11М3 применяется в качестве конструкционного материала для изготовления высокотемпературного оборудования и трубопроводов реакторных установок (РУ) на быстрых нейтронах с натриевым теплоносителем [1-2]. Особенностями эксплуатации стали в указанных элементах конструкций являются: работа в условиях ползучести и термического старения при заданном сроке службы не менее 30 лет. При этом работоспособность изделий на протяжении всего срока службы обеспечивается сохранением требуемого уровня механических свойств конструкционных материалов в условиях изменения структуры. Изменение структуры связано с выделением вторичных фаз элементов внедрения и замещения при температуре эксплуатации. Гарантией надежности обеспечения заданного срока службы с возможностью последующего его продления являются данные по изменению фазового состава материала в условиях эксплуатации и его влиянию на механические свойства. Фактическое состояние материала является определяющим при подтверждении результатов прогнозирования механических свойств и основным показателем возможности дальнейшей эксплуатации оборудования.

Цель настоящей работы - исследование изменений структуры и ее влияния на механические свойства стали марки 08X16Н11M3 после длительной высокотемпературной эксплуатации в составе теплообменного оборудования.

\section{2. Материалы и методика исследований}

Исследование изменений структуры и механических свойств выполнено на металле корпусов основного (ОП) и промежуточного (ПП) пароперегревателей - модулей парогенератора реакторной установки на быстрых нейтронах с натриевым теплоносителем после эксплуатации при температуре $515^{\circ} \mathrm{C}$ в течение 195000 ч. Химический состав исследованных материалов представлен в Табл. 1. В силу незначительных отличий металла ОП и ПП, в дальнейшем фактор химического состава исключается из рассмотрения и при анализе данных не учитывается.

Часть металла после эксплуатации была подвергнута термической обработке - аустенизации при температуре $1050^{\circ} \mathrm{C}$ (согласно нормативной документации на поставку). Далее состояние аустенизированного после эксплуатации металла будет рассматриваться в качестве исходного. Средние значения механических свойств стали марки 08X16Н11M3 в исходном состоянии представлены в Табл. 2.

Металлографические исследования проведены с использованием светового инвертированного оптического микроскопа Axio Observer. A1m Zeiss. Микроструктура изучалась на шлифах после электролитического травления в $10-\%$ растворе щавелевой кислоты.

Фазовый состав стали в равновесном состоянии определялся путём термодинамического моделирования [3] в программном пакете FactSage, совместно разработанном Thermfact/CRCT (Монреаль, Канада) и GTT-

Табл. 1. Химический состав исследованных фрагментов из стали марки 08Х16Н11M3.

Table 1. Chemical composition of the investigated fragments from steel grade 08Cr16Ni11Mo3 (grade 316).

\begin{tabular}{|c|c|c|c|c|c|c|c|c|}
\hline \multirow{2}{*}{ Модуль / Module } & \multicolumn{7}{|c|}{ Содержание элементов, мас.\% / Content of elements, mas.\% } \\
\cline { 2 - 9 } & $\mathrm{C}$ & $\mathrm{Cr}$ & $\mathrm{Ni}$ & $\mathrm{Si}$ & $\mathrm{S}$ & $\mathrm{Mo}$ & $\mathrm{P}$ & $\mathrm{Mn}$ \\
\hline ОП / MS & 0.07 & 16.0 & 10.02 & 0.48 & 0.007 & 2.12 & 0.014 & 1.27 \\
\hline ПП / RH & 0.07 & 16.0 & 10.02 & 0.48 & 0.007 & 2.21 & 0.014 & 1.27 \\
\hline ПП / RH & 0.08 & 16.23 & 10.2 & 0.59 & 0.01 & 2.22 & 0.015 & 1.41 \\
\hline
\end{tabular}

табл. 2. Средние значения механических свойств стали марки 08Х16Н11М3 в исходном состоянии.

Table 2. Average values of mechanical properties of steel grade $08 \mathrm{Cr} 16 \mathrm{Ni11Mo3}$ (grade 316) in the initial state.

\begin{tabular}{|c|c|c|c|}
\hline $\begin{array}{c}\text { Температура испытаний, }{ }^{\circ} \mathrm{C} \\
\text { Test temperature, }{ }^{\circ} \mathrm{C}\end{array}$ & $\begin{array}{c}\text { Временное сопротивление, МПа } \\
\text { Tensile strength, MPa }\end{array}$ & $\begin{array}{c}\text { Предел текучести, МПа } \\
\text { Yield strength, MPa }\end{array}$ & $\begin{array}{c}\text { Относительное сужение, \% } \\
\text { Reduction of area, } \%\end{array}$ \\
\hline 20 & 617 & 261 & 82.4 \\
\hline 350 & 493 & 186 & 62.3 \\
\hline 500 & 474 & 152 & 61.9 \\
\hline 550 & 441 & 178 & 69.0 \\
\hline 600 & 407 & 139 & 65.6 \\
\hline
\end{tabular}


Technologies (Аахен, Германия). Экспериментальные исследования фазового состава металла проведены с использованием сканирующего растрового электронного микроскопа Vega TESCAN, оснащенного рентгеновским энергодисперсионным спектрометром X-Maх-50, а также методом дифракции обратно рассеянных электронов (EBSD) с использованием растрового двулучевого электронно-ионного микроскопа Quanta 200 3D FEG.

Исследования механических свойств при испытаниях на статическое растяжение проводились на пятикратных образцах с диаметром рабочей части 6 мм по методике ГОСТ 1497-84 при комнатной температуре и ГОСТ 9651-84 при повышенной температуре.

\section{3. Экспериментальная часть}

Изменение структуры в процессе длительных тепловых выдержек материала, аустенизированного перед началом эксплуатации, обусловлено выделением из твердого раствора элементов с ограниченной растворимостью. Диффузионная подвижность указанных элементов при температуре эксплуатации инициирует процессы массопереноса и приводит к образованию вторичных фаз (Рис. 1). На Рис. 1a, b представлена структура стали 08X16Н11M3 после эксплуатации и в исходном состоянии соответственно. Структура стали после эксплуатации характеризуется большим количеством мелких выделений, локализованных в основном по границам зерен, которые по сравнению с исходным состоянием, обладают повышенной травимостью. В теле зерен после эксплуатации обнаружены отдельные выделения и двойники. При этом структура стали в исходном состоянии практически однофазная.

Химический состав, структура, размер, количество и локализация вторичных выделений относятся к основным факторам изменяющим механические свойства материала. Как показали результаты исследований с использованием электронной микроскопии в структуре стали после эксплуатации, кроме аустенита, обнаружены: карбид типа $\mathrm{M}_{23} \mathrm{C}_{6}(\mathrm{M}-\mathrm{Cr}, \mathrm{Mo})$, феррит (a), интерметаллидные фазы (Рис. 2). Следует отметить,

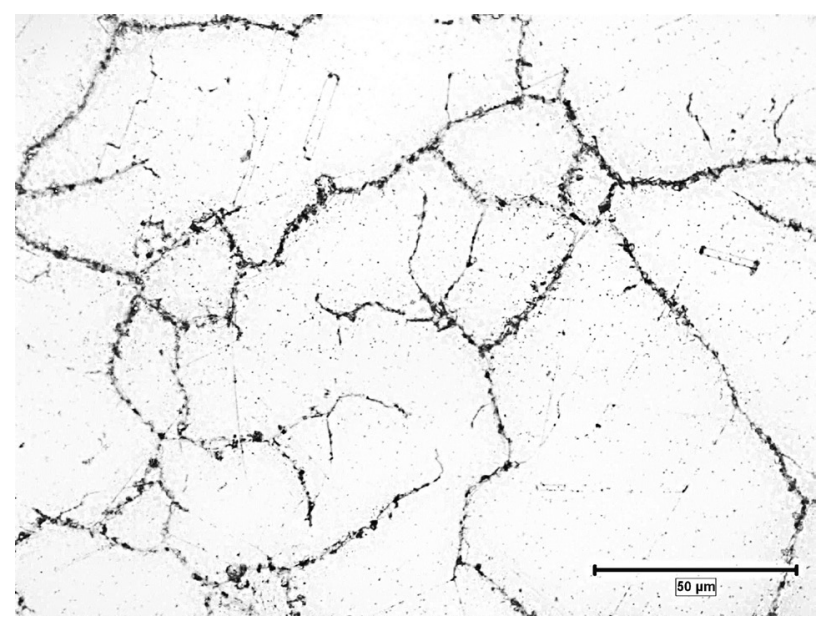

a

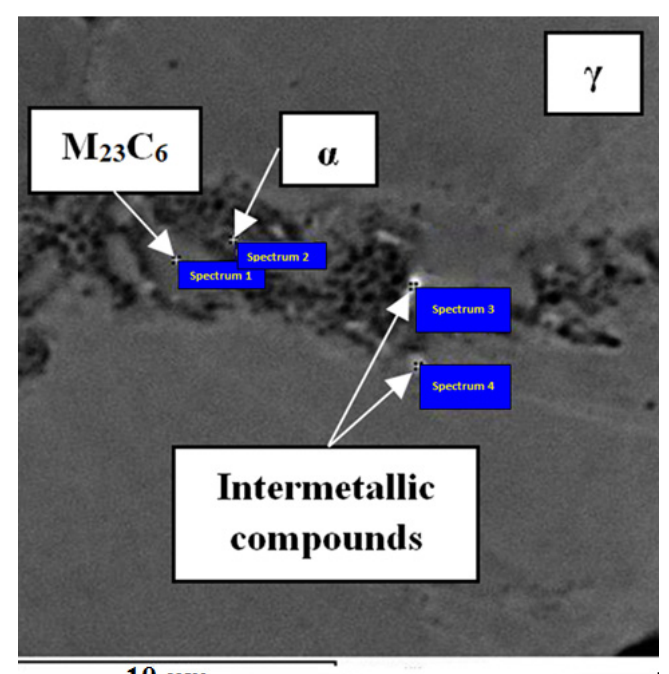

$10 \mu \mathrm{m}$

Рис. 2. РЭМ-структура стали марки 08X16Н11M3 после эксплуатации.

Fig. 2. SEM-structure of steel grade 08Cr16Ni11Mo3 (grade 316) after operation.

что интерметаллидные фазы, близки по химическому составу к фазе Лавеса, однако, в одной них содержание никеля значительно ниже. Идентификация указанных фаз проведена методом дифракции обратно рассеянных электронов (EBSD). Установлено, что вторичная фаза с низким содержанием никеля - $\chi$-фаза. Выделения локализованы в основном по границе зерна аустенита.

Обнаруженные вторичные выделения могут оказывать заметное влияние на механические свойства стали. Выделения карбидов и интерметаллидов могут приводить к значительному тепловому охрупчиванию, сопровождающемуся изменением механических свойств.

Выделения вторичных фаз в процессе эксплуатации образуются последовательно и являются взаимосвязанными. На первом этапе эволюции структуры стали, по причине ограниченной растворимости углерода в аустените при температуре эксплуатации, происходит выделение карбидов типа $\mathrm{M}_{23} \mathrm{C}_{6}$. Это приводит к формированию вблизи карбидных выделений участков,

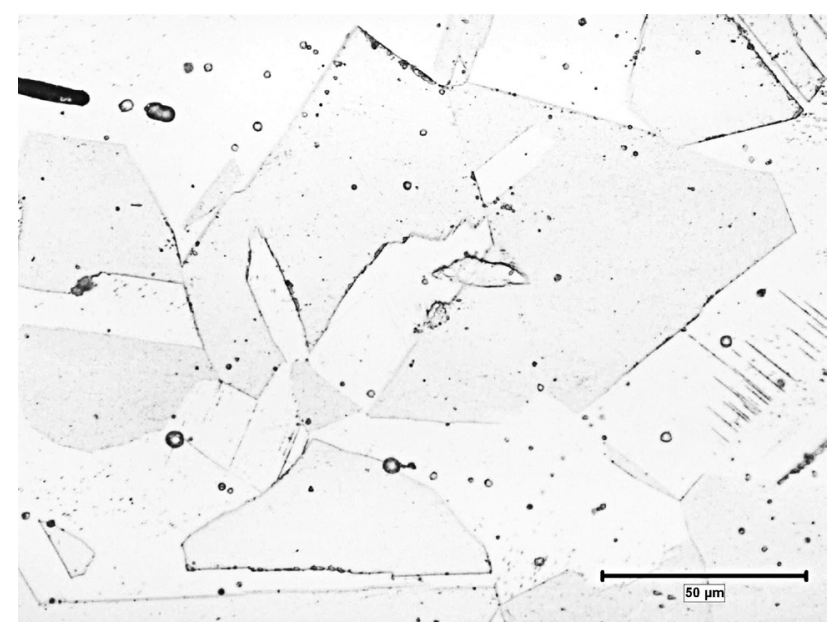

$\mathrm{b}$

Рис. 1. Микроструктура стали марки $08 \mathrm{X} 16 \mathrm{H} 11 \mathrm{M} 3$ после эксплуатации (а) и в исходном состоянии (b).

Fig. 1. Microstructure of steel grade $08 \mathrm{Cr} 16 \mathrm{Ni1} 1 \mathrm{Mo} 3$ (grade 316) after operation (a) and in the initial state (b). 
с пониженным содержанием хрома. Известно [4], что хром при содержании его в стали $\approx 17 \%$ в сочетании с никелем, способствует получению аустенитной структуры, как следствие сложного межатомного взаимодействия. Поэтому в зонах, обедненных хромом происходит превращение аустенита в феррит (a). Описанный механизм объясняет наблюдаемую на Рис. 2 локализацию вторичных выделений: к светлым участкам карбидов примыкают темные зоны феррита ( $\alpha$ ). Вблизи указанных областей формируются и частицы интерметаллидов, локализуясь по границам аустенитных зерен.

Достижение структурой состояния термодинамического равновесия при данной температуре приводит к окончанию процессов выделения вторичных фаз при дальнейшей выдержке (фазовый состав и объемная доля выделений будут оставаться неизменными). Термодинамическое моделирование показывает наличие $\sigma$-фазы в структуре стали при температуре эксплуатации $\left(515^{\circ} \mathrm{C}\right)$, что не подтверждено экспериментально, в то время как наличие обнаруженной $\chi$-фазы термодинамически не предсказано. То есть существует термодинамическая возможность протекания реакции образования б-фазы при дальнейшей эксплуатации (отсутствие $\chi$-фазы в равновесном состоянии связано с тем, что эта фаза является промежуточной и при увеличении времени выдержки в условиях соответствующих предпосылок будет претерпевать дальнейшую трансформацию, при этом возможно непосредственное превращение $\chi$-фазы в $\sigma$-фазу [5]. В остальном фазовый состав определенный экспериментально совпадает с результатами моделирования.

Механизм образования б-фазы до конца не ясен, согласно современным представлениям он связан с ферритом, $\chi$-фазой, карбидами $[5,6]$, при этом условием фазового перехода является повышенное содержание хрома [7]. По данным локального элементного анализа отношение содержания хрома в $\alpha$-феррите к содержанию хрома в аустените $(\gamma)$ составляет $\mathrm{Cr}^{\alpha} / \mathrm{Cr}^{\gamma}=0.52$, а по результатам термодинамического моделирования $\mathrm{Cr}^{\sigma} / \mathrm{Cr}^{\gamma}=11.32, \mathrm{Cr}^{\alpha} / \mathrm{Cr}^{\gamma}=3.66$, следовательно, предпосылок для образования $\sigma$-фазы в исследуемом металле нет. Следовательно, можно заключить, что в целом фазовый состав при дальнейшей эксплуатации не изменится. Полученные результаты по отсутствию в структуре $\sigma$-фазы хорошо согласуются с данным [7], где указано, что температурный интервал образования б-фазы в нержавеющих сталях составляет $600-1000^{\circ} \mathrm{C}$. Кроме того, количество б-фазы по данным работы [8] после тепловой выдержки стали марки 304 (аустенитная хромоникелевая сталь) при $700^{\circ} \mathrm{C}$ продолжительностью 100000 ч составляет $\approx 1.5 \%$.

О достижении объемной доли вторичных выделений равновесного значения можно судить по замедлению процесса теплового старения, вызванного изменениями структуры в процессе длительного воздействия высоких температур на сталь, например, по проявлению тепловой хрупкости [9]. Индикатором степени охрупчивания может служить, например, пластичность металла при комнатной температуре. На Рис. 3 представлена зави-

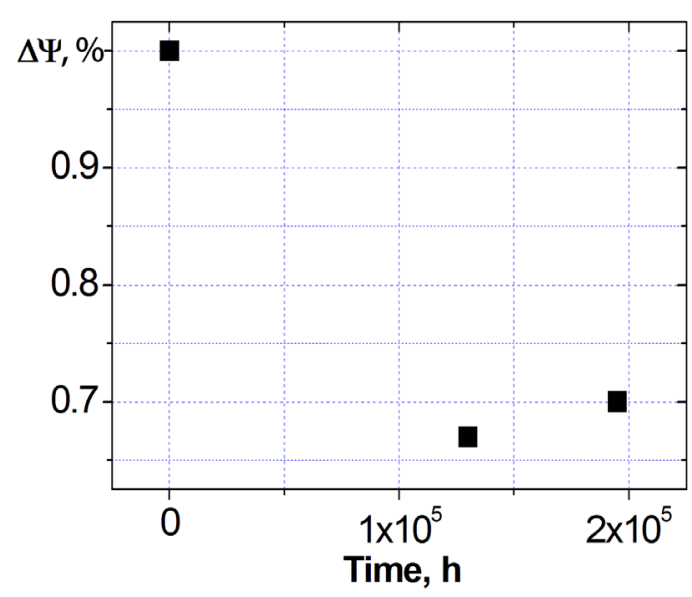

Рис. 3. Тепловое охрупчивание стали марки 08X16Н11M3 в процессе термического старения при температуре $515^{\circ} \mathrm{C}$.

Fig. 3. Thermal embrittlement of steel grade $08 \mathrm{Cr} 16 \mathrm{Ni1} 1 \mathrm{Mo} 3$ (grade 316) during thermal aging at a temperature $515^{\circ} \mathrm{C}$.

симость снижения относительного сужения, наблюдаемая при увеличении продолжительности тепловой выдержки $\left(\Delta \psi=\psi / \psi^{0}-\right.$ отношение значений сужения после термического старения при температуре $515^{\circ} \mathrm{C}$ к значениям сужения в исходном состоянии).

Охрупчивание стали в начальный момент времени связано с процессом образования карбидов, дальнейшее снижение пластических характеристик продолжается за счет выделения интерметаллидных фаз, так как инкубационный период их зарождения значительно выше, чем у карбидов [10]. После эксплуатации продолжительностью $\approx 130000$ ч процесс теплового охрупчивания резко замедляется. Стабилизация процесса теплового охрупчивания является признаком завершения процесса образования и роста вторичных карбидных и интерметаллидных фаз.

Изменения структуры после эксплуатации в течение 195000 ч при температуре $515^{\circ} \mathrm{C}$ оказывают влияние на прочностные характеристики стали (Рис. $4 \mathrm{a}$ ), $\Delta \sigma$ - отношение прочностной характеристики после эксплуатации к соответствующему значению в исходном состоянии). Временное сопротивление при комнатной температуре на 5\% выше исходного, а в диапазоне повышенных температур $350-600^{\circ} \mathrm{C}$ на 5-10\% ниже исходного уровня. Предел текучести при температурах 20,500 и $600^{\circ} \mathrm{C}$ после эксплуатации повышается на 5-10\% от исходного уровня, а при температурах 350 и $550^{\circ} \mathrm{C}$ практически не изменятся. Изменения прочностных характеристик связаны с перераспределением углерода, который выделяется из пересыщенного твердого раствора, образуя карбиды, а также с образованием интерметаллидных фаз по границам зерен. Эти процессы приводят к повышению сопротивления движению дислокаций за счет частиц вторичных фаз [11].

В процессе эксплуатации наблюдаются значительные изменения пластичности (Рис. $4 \mathrm{~b}$ ), $\Delta \psi-$ изменение значений относительного сужения стали в состоянии «после эксплуатации» относительно значений в исходном состоянии). Наибольшее снижение относительного сужения ( $\approx 30 \%)$ наблюдается при комнатной температуре. 


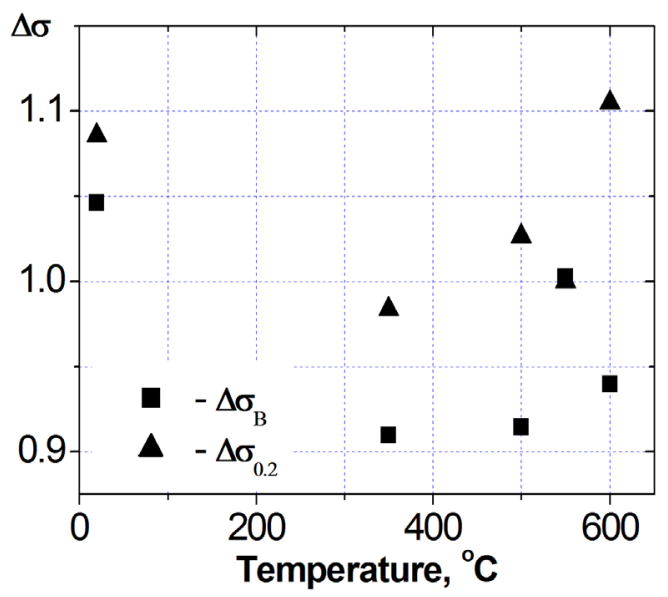

a

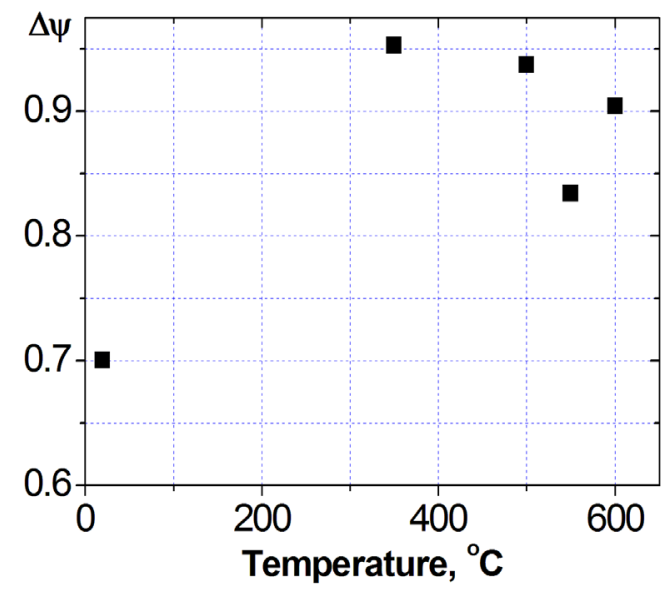

b

Рис. 4. Изменение механических свойств стали марки $08 \mathrm{X} 16 \mathrm{H} 11 \mathrm{M} 3$ после эксплуатации при температуре $515^{\circ} \mathrm{C}$ в течение 195000 ч: прочностные характеристики (a), относительное сужение (b).

Fig. 4. Changes in the mechanical properties of steel grade $08 \mathrm{Cr} 16 \mathrm{Ni} 11 \mathrm{Mo} 3$ (grade 316 ) after operation at a temperature $515^{\circ} \mathrm{C}$ for $195000 \mathrm{~h}$ : strength characteristics (a), reduction of area (b).

В диапазоне эксплуатационных температур значения пластичности стали марки 08X16Н11M3 на 5-17\% ниже исходного уровня. Снижение пластичности связано с наличием большого количества вторичных выделений карбидов и интерметаллидов, формирующихся в процессе эксплуатации преимущественно по границам зерен аустенита. Это приводит к повышению количества пор при пластической деформации вблизи границ зерен путем проскальзывания частиц второй фазы или отслаивания от матрицы. Рост пор усиливается локализацией пластической деформации. Поэтому пластичность стали снижается при увеличении объемной доли вторичных фаз [11].

\section{4. Выводы}

1. Эксплуатация продолжительностью 195000 ч при температуре $515^{\circ} \mathrm{C}$ приводит к изменению структуры стали марки 08X16H11M3, которое заключается в выделении карбидов, интерметаллидных фаз и феррита, преимущественно по границам зерен.

2. Структура стали марки 08X16Н11M3 близка к равновесной. Существуют термодинамические предпосылки к трансформации интерметаллидной $\chi$-фазы. Стабилизация процесса теплового охрупчивания свидетельствует о резком замедлении или прекращении процессов выделения и роста вторичных фаз.

3. Прочностные характеристики стали марки 08Х16Н11М3 в состоянии «после эксплуатации» близки к исходным. При этом в диапазоне температур испытаний $350-600^{\circ} \mathrm{C}$ наблюдается снижение пластичности на $5-17 \%$ от исходного уровня.

Благодарности/Acknowledgements. Экспериментальные исследования выполнены на оборудовании Центра коллективного пользования научным оборудованием «Состав, структура и свойства конструкиионных и функииональных материалов» НИЦ «Курчатовский институт» - ЦНИИ КМ «Прометей» при финансовой поддержке Министерства науки и высшего образования Российской Федераиии, соглашение 13.ЦКП.21.0014 (075-11-2021-068), уникальныцй идентификатор RF----2296.61321X0014. / Experimental studies were carried out on the equipment of the Core shared research facilities "Composition, structure and properties of structural and functional materials» of the NRC «Kurchatov Institute» - CRISM "Prometey» with the financial support of the state represented by the Ministry of Education and Science of the Russian Federation under agreement No. 13.CKP.21.0014 (075-11-2021-068). The unique identifier is RF----2296.61321X0014.

\section{Литература / References}

1. YU. F. Balandin, I.V. Gorynin, YU. I. Zvezdin, V.G. Markov. Structural materials for NPP. Moscow, Energoatomizdat (1984) 280 p. (in Russian) [Ю.Ф. Баландин, И.В. Горынин, Ю.И. Звездин, В.Г. Марков. Конструкционные материалы АЭС. Москва, Энергоатомиздат (1984) 280 с.]

2. G.P. Karzov, A.S. Kudryavcev, V.G. Markov, R.N. Grishmanovskaya, YU. M. Trapeznikov, M. A. Anan'eva. Material Science Issues. 2 (82), 23 (2015). (in Russian) [Г.П. Карзов, А.С. Кудрявцев, В. Г. Марков, Р.Н. Гришмановская, Ю. М. Трапезников, М. А. Ананьева. Вопросы материаловедения. 2 (82), 23 (2015).]

3. V.M. Golod, K.D. Savel'ev. Computational thermodynamics in materials science: study guide. SaintPetersburg, Polytechnic University Press (2010) 218 p. (in Russian) [В. М. Голод, К. Д. Савельев. Вычислительная термодинамика в материаловедении: учебное пособие. Санкт-Петербург, Издательство Политехнического университета (2010) 218 с.]

4. V.S. Mes'kin. Basics of steel alloying. Moscow, State scientific and technical publishing house of literature on ferrous and non-ferrous metallurgy (1959) 688 p. (in Russian) [В.С. Меськин. Основы легирования 
стали. Москва, Государственное научно-техническое издательство литературы по черной и цветной металлургии (1959) 688 с.]

5. K.H. Lo, C.H. Shek, J.K.L. Lai. Materials Science and Engineering R. 65, 39 (2009). Crossref

6. A.M. Parshin. Structure, strength and radiation damageability of corrosion-resistant steels and alloys. Chelyabinsk, Metallurgiya, Chelyabinsk branch (1988) 656 p. (in Russian) [A.M. Паршин. Структура, прочность и радиационная повреждаемость коррозионностойких сталей и сплавов. Челябинск, Металлургия, Челябинское отделение (1988) 656 с.]

7. C.-C. Hsieh, W. Wu. International Scholarly Research
Network ISRN Metallurgy. 2012, 732471 (2012). Crossref

8. T. Sourmail. Materials Science and Technology. 17 (1), 1 (2001). Crossref

9. G.P. Karzov, B.T. Timofeev, T.A. Chernaenko. Material Science Issues. 2, 42 (2005). (in Russian) [Г.П. Карзов, Б. Т. Тимофеев, Т.А. Чернаенко. Вопросы материаловедения 2, 42 (2005).]

10. A. F. Padilha et al. Journal of Nuclear Materials. 362, 132 (2007). Crossref

11. F. B. Pikering. Physical metallurgy and steel development. Moscow, Metallurgiya (1982) 184 p. (in Russian) [Ф.Б. Пикеринг. Физическое металловедение и разработка сталей. Москва, Металлургия (1982) 184 с.] 